\title{
VR Solutions for Improving Physical Therapy
}

\author{
Carlo Camporesi* \\ School of Engineering \\ University of California, \\ Merced
}

\author{
Marcelo Kallmann ${ }^{\dagger}$ \\ School of Engineering \\ University of California, \\ Merced
}

\author{
Jay J. Han \\ Department of Physical \\ Medicine and Rehabilitation \\ University of California, \\ Davis
}

\begin{abstract}
We present new solutions based on Virtual Reality technologies for improving the delivery of physical therapy and rehabilitation. Three main aspects are addressed: 1) the ability to allow therapists to create new exercises and therapy programs intuitively by direct demonstration, 2) automatic therapy delivery and monitoring with the use of an autonomous virtual tutor that can monitor and quantitatively assess the motions performed by the patient, and 3) networked collaborative remote therapy sessions via connected applications displaying the motions of both the therapist and the patient.

We also provide 3D assessment tools for monitoring changes in the range of motion, and for allowing the visualization of a number of properties during or after the execution of exercises. The presented system has been implemented for a low-cost hardware solution based on Kinect and for a high-end immersive virtual reality facility.
\end{abstract}

Index Terms: I.3.2 [Computing Methodologies]: Computer Graphics-Distributed/network graphics.

\section{INTRODUCTION}

Rehabilitation and physical therapy are optimal when assessment, monitoring, adherence to the therapy program and patient engagement can be achieved. With recent technical advances developed in Virtual Reality (VR), innovative approaches to improve traditional physical therapy and rehabilitation practice can be explored.

In traditional physical therapy and rehabilitation practice different processes are involved: physical examination, evaluation, assessment, therapy intervention, monitoring, and modification of the therapy program according to patient recovery. Usually, after a preliminary step of diagnostic and quantitative measurements, a patient is guided by a trained therapist to perform specific therapeutic exercises correctly. The tasks performed are designed according to the recovery plan and imply repetitions where the therapist needs to evaluate the exercise both qualitatively and quantitatively.

This process is usually intensive, time consuming, dependent on the expertise of the therapist, and implies the collaboration of the patient who is usually asked to perform the therapy multiple times at home with no supervision $[1,5]$.

Our solution allows therapists to model exercises by demonstration and thus to customize exercises for specific patient needs. Libraries of exercises can be developed for effective reuse in new therapy programs. Therapy programs can be performed by a virtual character demonstrating exercises step by step, including monitoring and logging patient execution. Monitoring and progress tracking improves patient understanding, motivation and compliance, and also provides data gathering. Finally, the system also allows simultaneous networked sessions between remote patients

\footnotetext{
*e-mail: ccamporesi@ucmerced.edu

†e-mail: mkallmann@ucmerced.edu

†e-mail: jay.han@ucdmc.ucdavis.edu
}
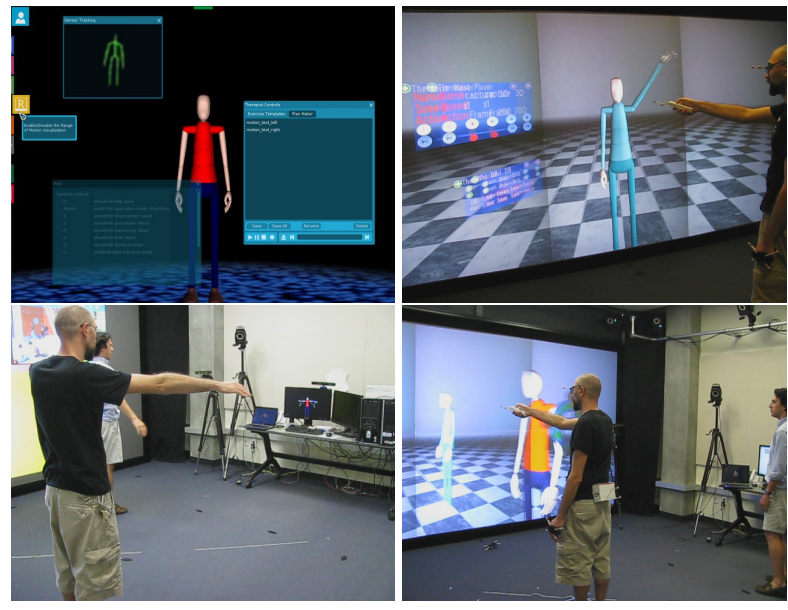

Figure 1: Our virtual collaborative system can run in two configurations: an inexpensive Kinect-based setup (left) and a high-end Immersive setup (right).

and therapists sharing motion performances in real-time. The system also provides 3D assessment tools for monitoring the range of motion, and for allowing the visualization of a number of therapy parameters during or after execution of exercises.

\section{SyStem IMPLEMENTATION}

The proposed system was implemented in two main configurations.

Immersive VR configuration: the main purpose of this configuration is to allow the therapist to immersively model customized exercises by demonstration and to experience high-end visualization of the performance of a patient. The patient's motion can be generated in real-time or it can be loaded from previously logged sessions. The application provides stereo visualization for enhanced comprehension of the observed motions and data. The user's upper body motions are tracked using a precise motion tracking system (based on Vicon cameras). For simpler setup, the system is configured to only track markers attached to the hands, torso and head. The motion is calibrated and mapped to the avatar following the same approach as described in the work of Camporesi et al. [3]. When connected to a remote site, two avatars are displayed for representing the connected patient and therapist. Previously recorded sessions can also be played on any of the avatars. The avatars can be visualized side-by-side or superimposed with transparency.

Our experimental immersive setup consists of a Powerwall system composed of six rendering computers, a main rendering node and an external computer driving the devices and the motion capture system. This choice provides a large immersive display that enhances user engagement allowing a better spatial understanding and analysis of motions. The interaction with the application is also fully immersive; thanks to virtual pointers and a 3D GUI interface controlled by a Wiimote (the GUI provides menus, buttons, generic widgets and panels). 
Kinect configuration: the second configuration is designed to assist patients when they perform their exercises. The patient is tracked through a non-cumbersome tracking device (for example, Microsoft Kinect or similar) and a virtual character (or virtual therapist) helps the patient perform the prescribed daily therapy tasks by providing real-time monitoring, feedback and logging. This configuration is also suitable for use at homes or clinics.

This configuration also provides two avatars when a networked connection is established. Even though the accuracy of Kinect is limited (and the accuracy drops when body occlusions occurs) it still provides a good tradeoff between cost and portability [2, 4]. Automatic motion detection mechanisms are provided to improve the usability of the system. For example, automatic display of joint angles only when significant variation is detected, end of exercise automatically detected after a period of inactivity, etc.

\subsection{Virtual Exercise Modeling, Delivery and Monitoring}

Both applications provide tools for modeling exercises and for delivery and monitoring of the exercises. The option of creating customized exercises by demonstration enables the therapist to go beyond recovery plans limited to a set of predefined exercises. Several interactive tools are available for assisting the therapist with creating new exercises by demonstration. The therapist can record demonstrations and then customize them in different ways. After a validation process the motions can be saved and categorized in a database of exercises. The database is then used for fast construction of therapy programs using a desktop-mode interface of the application during consultation with patients.

Both low-end and high-end configurations of the system include the exercise modeling by demonstration capability. Even though the two configurations offer similar capabilities, the accuracy of the employed sensor will lead to different limitations. The immersive configuration based on Vicon cameras is clearly more accurate for the modeling task. On the other hand, the versatility of the Kinect solution grants to the therapist more flexibility during a one to one session, and gives a markerless, portable, and inexpensive solution. Together both systems complement each other well during the different phases of long term therapy programs.

The system can be employed as a tool to deliver exercises to patients at home, and can be also used during clinical appointments to measure and investigate the performance of a patient. In all cases sessions can be logged and later re-loaded for analysis and progress assessment.

When delivering a patient's daily program, the virtual therapist can start the session by demonstrating the exercises to the patient. In a subsequent step, the user is asked to follow the exercises while the application is recording the sensed motion. If the motion is detected to be significantly different than the demonstrated exercise, the appropriate visual feedback is provided to the user for motivating an improved performance and for better understanding of the exercise. The level of expected compliance and repetitions until compliance can be personalized and defined by the therapist specifically for each patient.

Four types of feedback were developed in order to provide visual and quantitative information about the user motions (in realtime or off-line): visualization of end-effector trajectories; dynamic range of motion measurements; distances from the target exercise and joint angle measurements (virtual goniometer).

\subsection{Remote Therapist-Patient consultation}

The system also allow patients and therapists to interact remotely in any configuration, saving travel costs, potentially increasing access to health care, and allowing more frequent monitoring. The motion of each user participating to the virtual collaboration is mapped directly to each respective avatar, and the avatars can be superimposed with transparency or appear side-by-side in the applications.
The communication between two peers in a collaborative session is based on a client-server UDP communication schema with added packet ordering, guaranteed communication reliability and optional data compression. The server application, after accepting and validating an incoming connection, starts sending information of the avatar of the current user (sender) and waits the update of the client's avatar (receiver). For instance, if the therapist application is started as a server, the therapist's avatar becomes the active character in the communication and the second character, the patient's avatar, becomes a receiving entity. If the patient's application is started as the client, the sender entity becomes the character of the patient's application while the tutor/therapist becomes a receiving entity waiting for further updates. A per-joint activity mechanism for saving bandwidth has been implemented in order to handle communication between peers with limited bandwidth capabilities.

All feedback tools are available during a virtual collaboration session. The therapist can demonstrate exercises, analyze the patient motion, load preset exercises from the database, watch the patient's performances and even record a patient motion in real time.

\section{Discussion}

The presented system has been developed in close interaction with clinical evaluators and clinical translational researchers specializing in physical medicine and rehabilitation. The system addresses needs for the real working environment of therapists and as well develops novel capabilities such as networked sessions and customized exercise modeling. The developed system is currently being prepared for evaluation in clinical environments with patients. The evaluation will provide quantitative monitoring of therapy sessions, and will also asses general patient participation and engagement aspects to the prescribed therapies.

The system has been installed at the UC Davis Medical Center and many preliminary experiments have already taken place. The initial informal evaluations performed with the system have led to several discussions that shaped the overall system. In general, therapists evaluate the possibility of having exercises automatically delivered and the ability to monitor patients at home very valuable, and the current solution already addresses needs in their current professional practice. The options to model customized exercises and to perform remote sessions with patients are novel solutions that will require gradual experimentation and assessment

\section{ACKNOWLEDGEMENTS}

This work was partially supported by NSF award IIS-0915665 and by CITRIS grant number 128 .

\section{REFERENCES}

[1] A. P. T. Association. Guide to Physical Therapist Practice. Rev 2nd Ed. American Physical Therapy Association, Alexandria, VA, 1999.

[2] B. Bonnechere, B. Jansen, P. Salvia, H. Bouzahouene, L. Omelina, J. Cornelis, M. Rooze, and S. Van Sint Jan. What are the current limits of the kinect sensor? In proceeding of 9th International Conference on Disability, Virtual Reality and Associated Technologies, pages 287 294, 2012.

[3] C. Camporesi, Y. Huang, and M. Kallmann. Interactive motion modeling and parameterization by direct demonstration. In Proceedings of the 10th International Conference on Intelligent Virtual Agents (IVA), 2010.

[4] C. Chien-Yen, B. Lange, M. Zhang, S. Koenig, P. Requejo, N. Somboon, A. Sawchuk, and A. Rizzo. Towards pervasive physical rehabilitation using microsoft kinect. In International Conference on Pervasive Computing Technologies for Healthcare (PervasiveHealth), San Diego, CA, USA, May 2012

[5] C. Norkin. . Measurement of Joint Motion. A Guide to Goniometry. F.A. Davis Company, Philadelphia, PA, 2003. 